\title{
REMOTE SENSING OF METHANE EMISSIONS BY COMBINING OPTICAL SIMILITUDE ABSORPTION SPECTROSCOPY (OSAS) AND LIDAR
}

\author{
Sandrine Galtier ${ }^{1}$, Christophe Anselmo ${ }^{1}$, Jean-Yves Welschinger ${ }^{2}$, Jean-Pierre Cariou ${ }^{3}$, Jean- \\ François Sivignon $^{1}$, Alain Miffre ${ }^{1}$ and Patrick Rairoux ${ }^{1^{*}}$
}

${ }^{1}$ Institut Lumière Matière, UMR5306 Université Lyon 1-CNRS, Université de Lyon, 69622 Villeurbanne, France,patrick.rairoux@univ-lyon1.fr

${ }^{2}$ Institut Camille Jordan, UMR 5208 Université Lyon 1-CNRS, Université de Lyon, 69622 Villeurbanne cedex, France

${ }^{3}$ Leosphere France, 14-16 rue Jean Rostand, 91400 Orsay, France

\begin{abstract}
Monitoring the emission of gases is difficult to achieve in industrial sites and in environments presenting poor infrastructures. Hence, robust methodologies should be developed and coupled to Lidar technology to allow remote sensing of gas emission. OSAS is a new methodology to evaluate gas concentration emission from spectrally integrated differential absorption measurements. Proof of concept of OSAS-Lidar for $\mathrm{CH}_{4}$ emission monitoring is here presented.
\end{abstract}

\section{INTRODUCTION}

Lidar remote sensing has found a large interest in dedicated field studies on atmospheric trace gases and high concentrated atmospheric compounds for air quality control, industrial emission surveys and for long-term evaluation of climate change [1-3]. For this purpose, methodologies development and technical solutions are well documented in the literature [4, 5]. Among these methodologies, differential absorption Lidar (DIAL) and Raman scattering Lidar (RSL) are appropriate for atmospheric trace gases and water vapor active remote sensing. In the past fifteen years, improvements in laser technology and new methodology developments have been achieved, making DIAL and RSL a standard in the environment and climate survey [6]. However, due to the weakness of Raman scattering crosssections, the RSL-methodology is often limited to the remote sensing detection of high concentrated gases such as water vapor. Moreover, to achieve accurate DIAL measurements, a tunable laser, having a narrow spectral line width, is required, with a high spectral resolution detector. Hence, when applying the DIAL-methodology, limitations may occur when the spectral width of the emission laser does not match the target gas absorption line [7]. Conversely, when the absorption line width is in the same range as the laser spectral width (or even narrower than), effective absorption cross section should be taken into account. It follows that a measurement uncertainty is added as for the detection of greenhouse gases such as water vapor $\left(\mathrm{H}_{2} \mathrm{O}\right)$, methane $\left(\mathrm{CH}_{4}\right)$ or carbon dioxide $\left(\mathrm{CO}_{2}\right)[8]$ in the mid-infrared spectral region. In this case, other methodologies, based on a broadband emission laser and a high spectral resolution detector, have been successfully applied, as we formerly published [9].

In this contribution, a new methodology is proposed to remotely address atmospheric traces gases, with no particular consideration on the spectral width of the emission laser. It is based on coupling Lidar remote sensing with OSAS [10]. Our study underscores that OSAS-Lidar can overcome the possible mismatch between the spectral width of the laser emission and the TGabsorption line, which may occur for atmospheric greenhouse gases remote sensing. Pioneer work in this field has been published by $\mathrm{H}$. Edner et al. [11] On Hg-containing atmosphere. More recently, Thomas et al. [12] proposed a more appropriate approach to solve the problem of nonspectrally resolved absorption lidar measurements, which was the basis of OSASLidar. This contribution is organized as follows: in section 2, the OSAS-Lidar methodology is presented by highlighting the mathematical formalism. OSAS experimental verification is shown, followed by a proof of concept. This contribution ends with a discussion and proposes outlooks. 


\section{OSAS LIDAR METHODOLOGY}

\subsection{OSAS Principle}

In the OSAS-methodology [10], the power spectral density (PSD) of a broadband light source (i.e. broader than the spectral width of one or several molecular absorption lines) is set to be similar with the TG-gas absorption spectrum (the active channel), while a second PSD is set in a less absorbing spectral region (the reference channel). The OSAS-principle is shown in Fig. 1 when considering methane in its $2 v_{3}$ absorption band. The active channel $\mathrm{P}_{0 ; 1}(\lambda)$, which overlaps the Q-branch absorption lines, undergoes light absorption along the optical path, while the reference channel $\mathrm{P}_{0 ; 2}(\lambda)$ experiences a lower light extinction. Hence, two different signals, $S_{1}$ and $S_{2}$, are detected after light absorption.

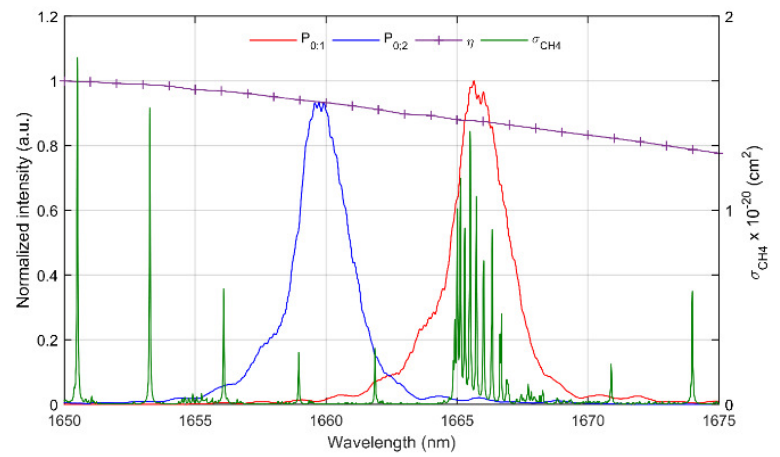

Figure 1: OSAS-principle applied to the $2 v_{3}$ Methane gas absorption band. The PSD of a broadband light source is spectrally shaped matching (red curve) or not (blue curve) the absorption cross-section spectrum $\sigma_{\mathrm{CH} 4}(\lambda)$ (green lines) In purple, the opto-electronic efficiency spectrum $\eta(\lambda)$ of the OSAS detection part.

\subsection{Target gas concentration retrieval}

The inversion algorithm used to retrieve the target gas concentration from spectrally-integrated OSAS-lidar signals $P_{1}(\mathrm{z})$ and $P_{2}(\mathrm{z})$ at altitude $\mathrm{z}$ is a particular application of what has been published in [10]. The starting point is the singlescattering elastic lidar equation:

$$
\begin{aligned}
P_{i}(z)=\frac{K}{z^{2}} \int_{\Delta \lambda} O(z, \lambda) P_{0 ; i}(\lambda) \beta(z, \lambda) \\
T_{T G}^{2}(z, \lambda) T_{\text {atm }}^{2}(z, \lambda) \eta(\lambda) \mathrm{d} \lambda
\end{aligned}
$$

where $O(z, \lambda)$ is the overlap function and $\beta(z, \lambda)$ the total backscattering coefficient. In the transmission term, the extinction from the target gas (TG) and from other atmospheric compounds are differently expressed, the latter being defined as follows at range $\mathrm{z}$ and wavelength $\lambda$ :

$$
T_{\text {atm }}(z, \lambda)=\exp \left[-\int_{0}^{z} \alpha\left(z^{\prime}, \lambda\right) \mathrm{d} z^{\prime}\right]
$$

The extinction coefficient $\alpha\left(z^{\prime}, \lambda\right)$ is the sum of four contributions, one per atmospheric main component (aerosols, molecules, except the target gas) considered in each physical process (light scattering, light absorption) [5]. TG-absorption is considered in the lidar equation by its transmission:

$$
T_{T G}(z, \lambda)=\exp \left[-\int_{0}^{z} \sigma_{T G}\left(z^{\prime}, \lambda\right) N_{T G}\left(z^{\prime}\right) \mathrm{d} z^{\prime}\right]
$$

Where $N_{T G}\left(\mathrm{z}^{\prime}\right)$ is the number density of the absorbing molecule of interest whose crosssection is $\sigma_{T G}$. Practically, the OSAS-lidar signals must - as in conventional lidar, be corrected from the sky background signal and electronic offsets. As in DIAL formalism, we made the assumptions that: (i) the overlap function $O(z)$ is the same for both wavelengths, (ii) the wavelength dependence of the extinction and backscatter coefficients $\alpha(z, \lambda)$ and $\beta(z, \lambda)$ of the atmosphere are negligible over the wavelength interval $\Delta \lambda$. In this way, the signals are modeled with the $f_{\mathrm{i}}$-functions, which are function of the path-integrated concentration or cumulative concentration parameter $x$ at distance $\mathrm{z}$ :

$$
f_{i}(z, x)=\int_{\Delta \lambda} P_{0 ; i}(\lambda) \exp \left[-2 \sigma_{T G}(\lambda) x(z)\right] \eta(\lambda) \mathrm{d} \lambda
$$

Following the formalism developed by Anselmo et al. [10], defining a g-function depending on $\mathrm{x}$ allows retrieving the cumulative concentration $C C(z)$ as follows:

$$
g(z, x)=S_{2} f_{1}(z, x)-S_{1} f_{2}(z, x)
$$

where $S_{i}$ are the lidar measurements. Because the $g$-function depends on the range $\mathrm{z}$, the cumulated concentration $C C(z)$ appears to be the first zero of the $g$-function. This zero value can be retrieved iteratively at each range $z$ by applying, as for example, the Newton-Raphson root-finding algorithm. Finally, the TG density number $N$ at 
range $z$ is retrieved by derivating $C C(z)$ with respect to $z$ :

$$
N_{T G}(z+\Delta z / 2)=\frac{\mathrm{d} C C(z)}{\mathrm{d} z}
$$

where $\Delta \mathrm{z}$ is the spatial resolution. Prior to applying the OSAS formalism, a verification that the ratio $f_{1}(x) / f_{2}(x)$ is monotonic on the concentration interval $\left[0, x_{\max }\right]$ should be made. Values of $x_{\max }$ can reach up to $10^{27} \mathrm{~mol} \mathrm{~m}^{-3}$ [10].

\section{OSAS-LIDAR SETUP}

This section presents the experimental arrangement of the OSAS-lidar methodology, built up to verify the proposed methodology for methane concentration retrievals. The general setup is presented in figure 2 . For this purpose, the emitter part is a OPO pumped fs Ti:Sapphire laser emitting in the NIR with a pulse repetition frequency of $1 \mathrm{kHz}$. The spectral switch between the active channel $\mathrm{P}_{0 ; 1}(\lambda)$ at $1665.5 \mathrm{~nm}$ and the reference channel $\mathrm{P}_{0: 2}(\lambda)$ at $1660 \mathrm{~nm}$ is achieved with an acousto-optic programmable dispersive filter. Laser pulses with energy of $30 \mu \mathrm{J}$ and with a $2 \mathrm{~nm}$ spectral width are achievable. As the laser amplitude modulation is performed at the emission, the lidar receiver configuration is quite simple. The light is collected with a Newtonian telescope having a focal length of $450 \mathrm{~mm}$ and a primary mirror diameter of $115 \mathrm{~mm}$. The NIR detector consists in an InGaAs based APD mounted at the telescope's focus point. The field of view $0.44 \mathrm{mrad}$ is governed by the $200 \mu \mathrm{m}$ detector diameter. The signal acquisition system is a 12 bits digitizing oscilloscope.

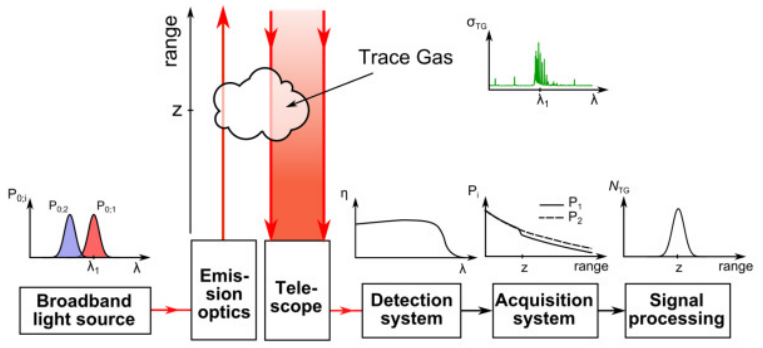

Figure 2: Principle of OSAS-lidar. Two different laser pulses are alternatively generated. Using a lidar system, these two emitted laser pulses through the atmosphere undergo different absorptions due to the $T G$ presence, giving rise to the output OSAS-lidar signals $S_{I}(z)$ and $S_{2}(z)$, after collection by a lidar receiver and detection on a photoreceptor.
For the first experimental achievement, a gas leak has been made artificially by using open gas cell located at a horizontal distance of 90 meters from the telescope. The open gas cell is 6 meters long with diameter of 60 centimeters. Hence, laser beam is able to pass through the gas cell without major interaction on his mount. To overcome the problem of low overlap between the receiver field-of-view and the laser transmitter in the near field, a biaxial geometry has been adopted.

\section{RESULTS}

The first lidar backscattering profiles with $30 \mu \mathrm{J}$ operating at $1665.5 \mathrm{~nm}$ for $\mathrm{P}_{1}$ and at $1660 \mathrm{~nm}$ for $\mathrm{P}_{2}$ are presented in Fig. 3. The strong and rapid signal increase observed at a $z=110$ meters distance is due to the reflection of a Teflon based hard target. The low signal increase at 90 meters range over a distance is due to the presence of particles injected in the open gas cell. Due to the turbulence highlighted by the fast particles concentration change (not visible in the figure), monitoring a $\mathrm{CH}_{4}$ with open path gas cell is found to be difficult within the signal to noise ratio related to fast acquisition. Then, TG concentration retrieval based on hard target reflection approach was therefore envisaged. The signal-to-noise ratio on the hard target reflection reach than $10^{3}$ (compared to $\mathrm{SNR}=2$ at $z=50 \mathrm{~m}$ ).

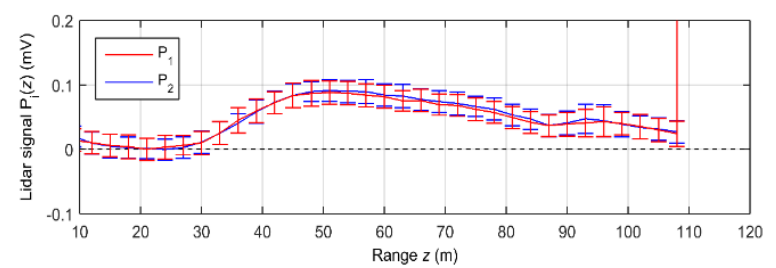

Figure 3: OSAS-Lidar signal for the active channel (red) and the reference channel (blue). The background noise signal is numerically subtracted. Each signal corresponds to a 1 second average time.

Remote Methane detection with OSAS-lidar is there achieved by filling out the open cell with air-methane. The first results on methane path integrated concentration (PIC) retrievals are presented in Fig. 4. When opening the gas cell after $\mathrm{CH}_{4}$ filling, the measurements show a methane PIC above $2.5 \times 10^{4}$ ppm.m. The dispersion of the methane plume contained into the open gas cell is very quick as it is expected. The results seem to be in agreement with the 
maximal value expected if we consider the $1.8 \%$ of methane mixing ratio released into a 6 meters long gas cell $\left(10.8 \times 10^{4} \mathrm{ppm} . \mathrm{m}\right)$. Since the gas cell is not perfectly sealed and its pressure remains at $1 \mathrm{~atm}$, a part of methane has necessarily leaked around during the filling.

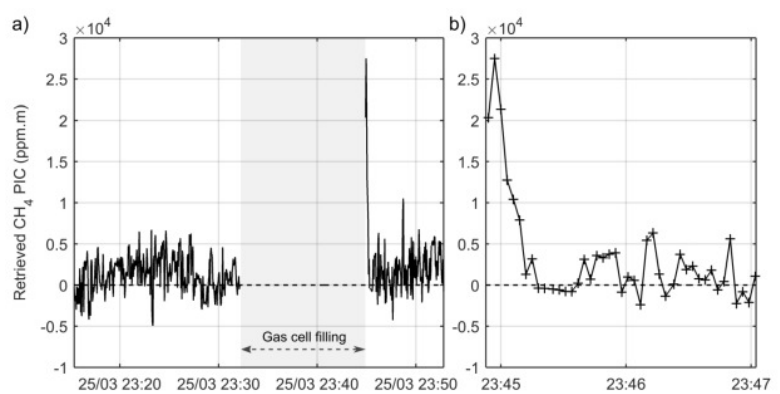

Figure 4: a) Retrieval of methane path integrated concentrations. The gas cell was closed during methane filling. b) Methane concentration dispersion. Once the gas cell opened, the methane concentration decreases quickly to the detection limit in less than 30 seconds.

An in-situ methane sensor placed within the open cell would have been very valuable to compare the two $\mathrm{CH}_{4}$ measurements. However, the detection limit $(1 \sigma)$ is estimated at $2.2 \times 10^{3}$ ppm.m.. As expected, this detection limit value is above the one obtained in laboratory $(\sigma=$ $0.65 \times 10^{3}$ ppm.m).

\section{CONCLUSION}

These preliminaries results appear promising though the estimated limit of detection is rather low. The possible outlooks of this work are numerous since these experimental results have been performed with a non-specifically dedicated laser light source. This work also settles the basements towards the use of the OSASmethodology to improve DIAL retrievals using broadband laser sources.

\section{References}

Numbered in the order they appear in the text, with brackets. Example of references:

[1] G. Ehret, C. Kiemle, M. Wirth, M. A. Amediek, A. Fix,S. Houweling, 2008: Spaceborne remote sensing of $\mathrm{CO}_{2}, \mathrm{CH}_{4}$, and $\mathrm{N}_{2} \mathrm{O}$ by integrated path differential absorption lidar: a sensitivity analysis, Appl. Phys. B, 90, 593-608.
[2] C. Stephan, M. Alpers, B. Millet, G. Ehret, P. Flamant, and C. Deniel, 2011: MERLIN: a space-based methane monitor, Proc. SPIE 8159, 815908.

[3] L. Fiorani, S. Santoro, S. Parracino, M. Nuvoli, C. Minopoli, and A. Aiuppa, 2015: Volcanic $\mathrm{CO}_{2}$ detection with a DFM/OPAbased lidar, Opt. Lett., 40, 1034-1036.

[4] R. M. Measures, 1992: Laser Remote Sensing, Fundamentals and applications, Krieger Ed.

[5] C. Weitkamp, 2005: Lidar, Range-Resolved Optical Remote Sensing of the Atmosphere, Springer Ed., Berlin, New-York.

[6] P. Di Girolamo, M. Cacciani, D. Summa1, A. Scoccione, B. De Rosa1, A. Behrendt, and V. Wulfmeyer, 2017: Characterisation of boundary layer turbulent processes by the Raman lidar BASIL in the frame of $\mathrm{HD}(\mathrm{CP}) 2$ Observational Prototype Experiment, $A C P$, 17, 745-767.

[7] V.D. Burlakov, 2010: A differentialabsorption lidar for ozone sensing in the upper atmosphere-lower stratosphere, Instruments and Experimental Techniques, 53, 886-889.

[8] S. Ismail et al., 2010: Laser measurements of water vapor, aerosol, and cloud distributions in Saharan air layers and tropical disturbances, J. Atmos. Sci., 67, 1026-1047.

[9] P. Rairoux et al. , 2000: Remote sensing of the atmosphere using ultrashort laser pulses, Appl. Phys. B, 71, 573-580.

[10] C. Anselmo, J.Y. Welschinger, J.P. Cariou, A. Miffre, P. Rairoux, 2016: Gas concentration measurement by optical similitude absorption spectroscopy: methodology and experimental demonstration, Opt. Expr., 24, 12588-12599.

[11] H. Edner, S. Svanberg, L. Unéus, W. Wendt, 1984: Gas correlation lidar, Opt. Lett., 9, 493.

[12] B. Thomas et al., 2013: Remote sensing of atmospheric gases with optical correlation spectroscopy and lidar: first experimental result on water vapor profile measurements, Applied Physics B, 113, 265-275. 